\title{
Zur Kenntniss des allgemein zu weiten Beckens.
}

\author{
Von \\ D $\circ \mathbf{h} \mathbf{n}$.
}

Während das enge Becken schon vielfach zum Gegenstande eingehender Untersuchungen gemacht worden ist, hat das $\mathrm{zu}$ weite Becken bisher noch keine entsprechende Beachtung erfahren. Es kann dies aufällig erscheinen, zwar nicht aus praktischen Rücksichten, aber doch aus wissenschaftlichem Interesse, denn es lässt sich voraussetzen, dass die Untersuchung des Wachsthums der einzelnen Theile des Beckenringes dasselbe Interesse darbietet in den Fällen, in welchen die Weite des Beckens die Norm übersteigt, als in denen, in welchen sie hinter dem Normalmaasse zurückgeblieben ist.

Durch die trefflichen Untersuchungen von Litzmann ${ }^{1}$ ) sind wir bezüglich des allgemein gleichmässig verengten Beckens dahin belehrt worden, dass es sich bei dieser Beckenform keineswegs um eine gleichmässige Verjüngung des Normalbeckens handelt, sondern dass einzelne Theile des Beckenringes mehr als andere in dem Wachsthum zurückgeblieben sind und dass in mehreren wichtigen Beziehungen jene Beckengattung an das kindliche Becken erinnert. Gegenüber diesem Resultate, dessen Richtigkeit späterhin noch durch ausgedehntere Messungen von einem meiner Schüler Bestätigung gefunden hat ${ }^{2}$ ), ergiebt sich auch für das gleichmässig zu weite Becken die Frage, wie sich denn hier jene Beckentheile verhalten, welche bei dem gleichmässig zu engen Becken in der Entwickelung zurückgeblieben sind.

1) Die Formen des Beckens. 1861.

2) Ries, H., Zur Kenntniss des allgemein gleichmässig verengten Beckens. Dissertation. Marburg 1868. 
Um hierüber Aufschluss zu erreichen, habe ich an zehn skelettirten allgemein zu weiten Becken entsprechende Messungen vorgenommen. Nicht alle von diesen Beckenpräparaten zeigten in strengem Sinne des Wortes die normale Beckenform, immerhin aber waren die beobachteten Abweichungen doch so gering, dass man die Mehrzahl derselben nicht nur als allgemein, sondern auch als gleichmässig zu weite Becken bezeichnen darf.

Die Ergebnisse dieser Messungen, bei deren Vornahme ich mich im Wesentlichen an die von Litzmann aufgestellten Rubriken gehalten habe, stelle ich in Folgendem zusammen:

\begin{tabular}{|c|c|c|c|c|c|c|c|c|c|c|c|}
\hline $\mathrm{Bec}$ & 1 & 2 & 3 & 4 & 5 & 6 & 7 & 8 & 9 & 10 & 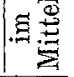 \\
\hline $\begin{array}{l}\text { reite der } \\
\text { gerader }]\end{array}$ & 14 & 11,1 & 11,9 & 12,0 & 12,1 & 12.7 & \begin{tabular}{|l|}
11,2 \\
11
\end{tabular} & 12,0 & 13,0 & 12,1 & 2 \\
\hline reite des $\mathrm{e}$ & 3.2 & 2,8 & 3,0 & 2,6 & 3,0 & 3,4 & 3,1 & $\begin{array}{r}3,0 \\
\end{array}$ & 3,2 & $\begin{array}{r}2,9 \\
\end{array}$ & \\
\hline Virbels & 5,4 & 4,1 & 4,4 & 4,7 & 4,6 & 4,7 & 4,0 & 4,5 & 4,9 & 4,5 & 4, \\
\hline $\begin{array}{l}\text { horium zur Spitze } \\
\text { in gerader Linie }\end{array}$ & 10,2 & 10,1 & 9,5 & 9,5 & 10,3 & 11,3 & 10,0 & 9,8 & 10,8 & 10,0 & 10 \\
\hline $\begin{array}{l}\text { osterior superior } \\
\text { on oberen Winkel } \\
\text { ularis }\end{array}$ & & 5,2 & 6,9 & $7 ; 2$ & 7,3 & & & & & & \\
\hline $\begin{array}{l}\text { om vorderen oberen Winkel der } \\
\text { Facies auricularis zum Tubercu- }\end{array}$ & 0,0 & 0,2 & $0, \mathfrak{Y}$ & $x, z$ & 7,3 & 8 & 8 & 官 & 7,9 & 6,4 & \\
\hline $\operatorname{lun}$ & 8,0 & 8,0 & 8,5 & 8,0 & 7,9 & 7,9 & 9,0 & 7,2 & 6,8 & 8,0 & 7 \\
\hline $\begin{array}{l}\text { Randes der Sym- } \\
\text { and des kleinen }\end{array}$ & 9,0 & 7,8 & 7,4 & 8,0 & 8,0 & 8,9 & 7,0 & 8,0 & 8,5 & 8,2 & 8 \\
\hline & 9,9 & 9,8 & 0,0 & 0,1 & 0,0 & 0,3 & 9,7 & 9,5 & 9,4 & 9,6 & \\
\hline pubis & 4,5 & 3,4 & 4,0 & 4,4 & 4,0 & 4,3 & 4,0 & 4,2 & 4,9 & 3,9 & 4 \\
\hline einhälfte & $102^{0}$ & $5^{0}$ & 1 & - & 115 & $8^{\circ}$ & $110^{\circ}$ & $121^{\circ}$ & - & - & 108 \\
\hline & $90^{\circ}$ & 118: & 10 & $92^{\circ}$ & $85^{\circ}$ & $95^{\circ}$ & 93 & $82^{\circ}$ & 90 & $115^{c}$ & 98 \\
\hline $\begin{array}{l}\text { vera mit der } \\
\text { vera mit dem }\end{array}$ & $98^{\circ}$ & $100^{\circ}$ & $108^{\circ}$ & $89^{\circ}$ & $90^{\circ}$ & $105^{0}$ & $92^{\circ}$ & $94^{\circ}$ & $95^{\circ}$ & $100^{\circ}$ & \\
\hline vera mit dem & $128^{\circ}$ & $122^{\circ}$ & E. & $103^{\circ}$ & - & $145^{\circ}$ & $100^{\circ}$ & $138^{\circ}$ & $142^{\circ}$ & & 125 \\
\hline sup. oss. ilei & 27,2 & 24,4 & 24,6 & 26,7 & 24,5 & 29,7 & 24,0 & 24,8 & 27,4 & 27,0 & 26 \\
\hline ilei & 28,9 & 27,0 & 27,3 & 29,2 & 29,0 & $30, \tilde{a}$ & 28,0 & 30,1 & 29,9 & 29,7 & 28 \\
\hline - & 7,2 & 9,1 & 8,0 & 8,4 & 7,7 & 8,0 & 7,4 & 7,5 & 7,0 & 8,5 & \\
\hline & 1 , & 1. & & & & & 1, & & 1 , & & \\
\hline Höl & 8,6 & 9 & 5 & & 8,2 & 9.5 & 8, & 7 , & 8,7 & 8,3 & \\
\hline es & & & & & 4,2 & & & 9 & 3,5 & 3,9 & \\
\hline & 1 & & & & 12,4 & 2 & 19 & 11,2 & 12,2 & 12 & 12 \\
\hline 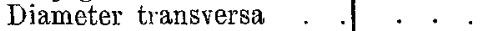 & & & & & 0 &, 0 & 4,0 &, 0 & 3,0 & & 14 \\
\hline & & 2 & & & & 1 & 13,5 & & 14,1 & 3,5 & 10 \\
\hline 0 -pect eingang & 16 & & 14 & 14,2 & 13,5 & & 12,4 & 14,2 & 14,0 & 14,0 & 14 \\
\hline loidea) & 0 & & & 2 & & & & & 6,2 & & \\
\hline - & & 13,2 & & & & & 14,2 & 13,3 & 1,1 & & 13 \\
\hline or transversa. & 1 & 13,4 & 14,2 & 14,5 & 13,0 & 14,2 & 14,0 & 14,9 & 14,5 & 14,0 & 14 \\
\hline istanta spinar. ischii & & 11,5 & 12,0 & 12,1 & 10,3 & 10,0 & 11,3 & 11,3 & 11,0 & 10,8 & 11 , \\
\hline
\end{tabular}




\begin{tabular}{l|l|c|c|c|c|c|c|c|c|c|c|c|c|c|}
\hline Becken Nr. & 1 & 2 & 3 & 4 & 5 & 6 & 7 & 8 & 9 & 10 & . \\
\hline
\end{tabular}

Ueberblickt man die vorstehenden Zahlwerthe, so erkennt man, dass auch hier wieder verschiedene Theile des Beckenringes eine gleichmässige Veränderung nicht erfahren haben, sondern dass einzelne mehr als andere über die Norm hinaus gewachsen sind. Es wird das am besten ersichtlich, wenn man diese Zahlen mit den von Litzmann und $\mathrm{Ri}$ es für das normale und das gleichmässig verengte Becken angegebenen Werthen vergleicht. Setzt man die Conjugata vera $=1$, so ergeben sich für die Durchmesser des kleinen Beckens folgende Werthe:

\begin{tabular}{|c|c|c|c|c|c|}
\hline & & & $\begin{array}{l}\text { Gleichmäs- } \\
\text { sig vereng- } \\
\text { tes Bocken }\end{array}$ & $\begin{array}{c}\text { Normales } \\
\text { Beeken }\end{array}$ & $\begin{array}{l}\text { Allgemein } \\
\text { zu weites } \\
\text { Becken }\end{array}$ \\
\hline Conjugata vera & . . & . & 1,00 & 1,00 & 1,00 \\
\hline Decken Diameter transversa & & . & 1,28 & 1,29 & 1,21 \\
\hline mang Diameter obliqua & . & & 1,21 & 1,20 & 1,15 \\
\hline Becken- f Diameter recta & . & . & 1,16 & 1,19 & 1,08 \\
\hline höhle Diameter transversa & . & & 1,11 & 1,15 & 1,21 \\
\hline Beckenausgang: Diameter tran & sversa. & & 1,06 & 1,15 & 0,98 \\
\hline
\end{tabular}

An der Bildung der vorstehenden Durchmesser betheiligen sich bei dem allgemein zu weiten Becken einzelne Beckentheile in einer von dem normalen und noch mehr von dem gleichmässig verengten Becken differirenden Weise. Vergleichen wir nämlich auch hier wieder mit den Litzmann'schen Angaben die unserigen, so ergiebt sich Folgendes:

Es verhält sich der Abstand der Spinae ilei posteriores superiores zu dem der Spinae ilei anteriores superiores wie

$1: 2,9$ beim gleichmässig verengten Becken,

$1: 3,0$, normalen Becken,

$1: 3,3 \quad, \quad$ allgemein $\mathrm{zu}$ weiten Becken.

Es verhält sich die Breite des Kreuzbeinflügels zur Breite des ersten Wirbelkörpers wie 
$1: 1,6$ beim gleichmässig verengten Becken,

$1: 1,3$, normalen Becken,

$1: 0,6$, allgemein zu weiten Becken.

Es verhält sich der Abstand der Spinae posteriores ilei zur Breite der Kreuzbeinbasis und zum Abstande der Tubercula ileopectinea wie

$1: 1,41: 1,52$ beim gleichmässig verengten Becken,

$1: 1,49: 1,56 \quad$, normalen Becken,

$1: 1,56: 1,80$, , allgemein zu weiten Becken.

Es beträgt der Winkel der Conjugata vera mit der vorderen Beckenwand im Mittel

$106,6^{\circ}$ beim gleichmässig verengten Becken,

99,4 ", normalen Becken,

97,1 , allgemein zu weiten Becken.

Wir sehen demnach an der Zusammenstellung der vorstehenden Zahlwerthe, dass in einzelnen wichtigen Beziehungen, welche Litzmann als charakteristisch für das gleichmässig verengte Becken hinstellt, das allgemein zu weite Becken geradezu entgegengesetzte Werthe aufweist, dass demnach in dem Wachsthume der betreffenden Beckentheile gewissermaassen eine Stufenleiter zu erkennen ist von dem gleichmässig verengten zu dem normalen und von diesem wieder zu dem allgemein zu weiten Becken.

Es ist sehr wohl möglich, dass dieses interessante Verhältniss sich auch noch für andere Beckentheile als für die angeführten weiter nachweisen lässt. Es wäre eine dankbare Aufgabe, durch die Ausmessung einer grösseren Anzahl ron Beckenpräparaten diesen Gedanken weiter zu verfolgen. 